\title{
1 A bite force database of 654 insect species
}

\section{Authors}

3 Peter Thomas Rühr, Carina Edel, Melina Frenzel, Alexander Blanke

4

5 Affiliation

6 Institute of Evolutionary Biology and Animal Ecology, University of Bonn, An der Immenburg

$7 \quad$ 1, 53121 Bonn, Germany

8

9 corresponding author: Peter T. Rühr (ruehr@uni-bonn.de) 


\section{Abstract}

11 Bite force is a decisive performance trait in animals because it plays a role for numerous life

12 history components such as food consumption, inter- and intraspecific interactions, and

13 reproductive success. Bite force has been studied across a wide range of vertebrate species,

14 but only for 20 species of insects, the most speciose animal lineage. Here we present the insect

15 bite force database with bite force measurements for 654 insect species covering 111 families

16 and 13 orders with body lengths ranging from $4.2-180.1 \mathrm{~mm}$. In total we recorded 1906 bite

17 force series from 1290 specimens, and, in addition, present basal head, body, and wing metrics. As such, the database will facilitate a wide range of studies on the characteristics, predictors, and macroevolution of bite force in the largest clade of the animal kingdom and to bite force across all biting metazoans. 


\section{Background \& Summary}

24 Bite force is a performance trait which may decide on an animal's ability to acquire food, win

25 inter- and intra-specific fights and successfully reproduce ${ }^{1-5}$. In vertebrates, maximum bite

26 forces are well studied across a wide diversity of taxa such as bony fishes ${ }^{6,7}$, crocodilians (e.g.

$27{ }^{8}$ ), birds (e.g. ${ }^{9}$ ), turtles (e.g. ${ }^{10}$ ), squamates (e.g. ${ }^{11,12}$ ), frogs ${ }^{13}$, marsupials ${ }^{14}$, and mammals (e.g. $\left.288^{15-18}\right)$.

29 Fundamental knowledge on the variation, predictors and evolution of bite forces within the 30 omnipresent insects is, however, lacking. Even though more than half a million insect species 31 belong to orders that possess biting-chewing mouthparts ${ }^{19,20}$, existing literature only yields bite force measurements on five dragonflies ${ }^{21,22}$, one cockroach ${ }^{23}$, and 14 beetles ${ }^{24,25}$. This is despite the fact that biting-chewing insects include the most destructive plant eating animals and occupy crucial roles in the world's ecosystems as soil-building detritivores ${ }^{26}$.

So far, measuring bite forces of insects was hampered by their small size, but the recently published measurement setup "forceX" 27 overcame this limitation to some extent by allowing minimally invasive in vivo bite force measurements of animals with gape sizes more than ten times smaller than previous setups (e.g. ${ }^{28}$ ). Using forceX, we measured bite forces of 654 insect species from 111 families and 13 orders, collected on four continents and from numerous breeding cultures. Instead of gathering maximum force values only, as most previous bite force studies have done (but see ${ }^{25,23,21,22,29}$ ), we also recorded force curves. In addition, the bite force database contains head, wing and body metrics of each specimen to assess morphological predictors for bite force in insects. Thus, the database will facilitate investigations on the macroevolution of maximum bite force, bite lengths, bite frequencies, muscle activation patterns, and bite curve shapes across the megadiverse insects and will facilitate comparisons with all biting metazoan taxa. 


\section{Methods}

\section{Collection and material}

A total of 1290 insect specimens representing 654 species from 111 families and 13 orders were collected in Australia, China, Denmark, France, Germany, Greece, Panama and Slovenia using light traps, insect nets, pitfall traps, or directly by hand. All specimens were collected under the respective regulations in effect (see Acknowledgements). Additionally, we measured specimens from numerous scientific, private and commercial insect breeders and traders (Online-only Table 1 and Acknowledgements).

\section{Size measurements}

Head width, head length, head height, thorax width, forewing length and body length measurements were performed to the nearest $0.01 \mathrm{~mm}$ using a digital caliper (77001, Wentronic $\mathrm{GmbH}$, Braunschweig, Germany). For the head width, the longest distance from left to right was measured, including protruding eyes if applicable (Fig. 1a). Head height in orthognathous insects was measured from the clypeo-labral ridge to the dorsal end of the head (Fig. 1a,b). In prognathous insects, head length was measured from the clypeo-labral ridge to the posterior end of the head (Fig. 1c). Thorax width was measured on the prothorax (Fig. 1d) and excluded lateral protrusions as found e.g. in many cockroaches and praying mantises. Body length measurements excluded cerci, ovipositors, or other abdominal appendages (Fig. 1e).

\section{Bite force measurements}

7 All measurements were carried out with the metal-turned version of the forceX setup as described in ${ }^{27}$. In short, life and conscious animals were held between two fingers, rotated by $90^{\circ}$ along their body axis and allowed to voluntarily bite on the tip elements of the forceX. 
Different tip element designs ${ }^{27}$ and distances between them were used to accommodate different animal gape sizes. During measurements, animals were observed through the Junior Stereo 3D microscope (Bresser $\mathrm{GmbH}$, Rhede, Germany) that is part of the forceX setup to ensure that gape sizes are suitable and that the insects bite at the edge of the tip elements so that the ratio of the forceX lever remains at a constant $0.538^{27,30}$. We also checked if the animals bit with the distal-most incisivi of their mandibles to ensure that measurements remain comparable ${ }^{30}$. Non-distal bites or wrongly placed bites on the tip elements were discarded. If animals did not start biting by themselves, we used the tip element protrusions to insert the tip elements between the mandibles and/or used a fine brush to touch the animal's cerci, head or abdomen ${ }^{23}$. Amplified analogue voltage signals were converted to a digital signal by a 12-bit USB data acquisition device (U3-HV, LabJack Corporation, Lakewood, Colorado, US) and recorded with the LStreamUD v1.19 measurement software (LabJack Corporation) on a computer.

\section{Data curation}

Subsequent data curation was performed in the software environment ' $R$ ' $v .4 .03^{31}$ using the package 'forceR' $v \cdot 1 \cdot 0 \cdot 0^{27}$. Since the forceR package was written to analyse data generated with the forceX setup, we used, if not stated otherwise, the default settings of the package functions. First, time series were converted from the output format of LStreamUD to a *.csv file containing only a time and a voltage column (without changing measurement values) using the forceR function 'convert_measurement()'. Then, all measurements were manually cropped using 'crop_measurement()' to exclude regions without bite data at the beginning and end of each measurement. Next, 'amp_drift_corr()' was used to correct for the logarithmic drift of the analogue charge amplifier (see Rühr and Blanke ${ }^{27}$ for details). When using the high amplification setting $(20 \mathrm{~V} / \mathrm{N})$ to amplify the miniscule voltage signals of the piezoelectric force transducer at small bite forces, the zero-voltage-line ('baseline') may drift notably during a 
measurement. Therefore, a PDF file depicting all input raw data and their amplifier driftcorrected data graphs (available at Zenodo, s. Data Records) was visually inspected, and, if necessary, the function 'baseline_corr()' was used in its automatic mode to correct for this drift. In some of these cases, however, especially when the test animals showed long, plateaulike bite curve shapes, the automatic mode of 'baseline_corr()' failed to find the baseline, and the manual mode was used. All corrections can be retraced in the PDF file and reproduced using the log files that were created during corrections and which are stored at Zenodo. With the function 'reduce_frq()' we then reduced the sampling rate of all time series to $200 \mathrm{~Hz}$, a value found to be sufficient to represent insect bite force curves ${ }^{25,23,22,22}$ to reduce the amount of data for further analyses. As a last curating step, voltage values were converted into force data [N] with the forceR function ' $y \_$to_force()' that considers the amplification level of each measurement and the lever mechanics of the measurement system. Online-only Table 1 shows all measurement settings, taxonomic classifications and information on which correction procedures have been performed on which measurements.

\section{Maximum force value extraction for specimens and species}

To extract maximum force values of each specimen and each species and calculate the standard deviations of these values we used the function 'summarize_measurements()' of forceR and custom code, heavily on the packages 'dplyr' v.1.0.7 ${ }^{32}$. We then plotted the log10transformed average maximum bite force per specimen (grey dots in Fig. 2) and per species (black dots) against the log10-transformed average body length (Fig. 2a) and head width (Fig. 2b) using 'ggplot2' v.3.3.5 $5^{33}$ and 'ggExtra' v.0.934. Linear regressions through the log10transformed species-wise data showed significant allometric relationships between body size and head width $(p<0.001)$ with explanatory values of $r^{2}=0.43$ and $r^{2}=0.56$, respectively. Due to the expected logarithmic releationship between size and bite force ${ }^{35}$, means were 
119 calculated as geometric means. Calculations with the regular mean, however, yielded similar

120 results $\left(p<0.001, r^{2}=0.44\right.$ and $r^{2}=0.56$; Supplementary Figure 1$)$.

121 Comparison to previous insect bite force measurements

122 Previous studies on insect bite forces covered maximum bite force values for 20

123 species ${ }^{24,23,21,22}$. To check if these measurements follow similar allometric slopes as our data,

124 we extracted all available insect bite force data from the literature and added them to the

125 scatterplot in Figure $2 \mathrm{~b}$. We then tested if our data and the literature data differ in their

126 allometric slopes by comparing a linear model with the null hypothesis of different slopes

$127(\log 10$ (bite.force) $\sim \log 10$ (head.width) * source) versus a linear model with the null hypothesis

128 of common slopes (log10(bite.force) $\sim \log 10$ (head.width) + source). Both model fits were

129 compared with an ANOVA to find out if they differ significantly.

130 Assessment of geographical coverage

131 Climate zone data (Köppen-Geiger classification system ${ }^{36-38}$ ) was gathered for each species

132 based on the GPS coordinates of its collection localities (Online-only Table 1) with the function

133 'LookupCZ()' of the R package 'kgc' v.1.0.0.2 ${ }^{39}$. Percentages of species in the database for each

134 country and climate zone were calculated.

\section{Assessment of phylogenetic coverage}

136 To assess the phylogenetic coverage of the bite force database we compared the number of

137 species with database entries to the number of species listed by the Open Tree of Life ${ }^{40}$,

138 accessed on 2022/02/05 with the function 'tol_node_info()' of the package 'rotl' v.3.0.11 ${ }^{41}$.

139 Comparisons were carried out for all insect orders and families that are present in the bite

140 force database. 


\section{Data Records}

142 All raw measurements, the cleaned time series, and the PDF and log files created during the 143 conversion of the raw data to the final database are available in comma-separated format at 144 Zenodo: https://doi.org/10.5281/zenodo.5782922). Online-only table 1 is also stored in the 145 same repository.

\section{Technical Validation}

147 Visual inspection of the scatter plot of bite force against head width (black dots in Fig. 2b) and

148 all literature data points (orange diamonds) revealed that the literature data lies close to the 149 regression through all data points of our database. This impression is corroborated by the 150 comparison of the allometric slopes of the insect bite force database and the literature data,

151 which yielded no statistically significant difference (ANOVA: $F=0.102, p=0.75$ ).

152 Geographical assessment of the collected animals showed that most species of the insect bite 153 force database were collected in Australia (30.7\%), Germany (19.1\%), and Panama (16.4\%).

$15423.2 \%$ of the species were obtained from breeding cultures. The remaining $10.6 \%$ of the 155 species were collected in Greece, Slovenia, France, China, and Denmark. Climate region 156 assessment revealed that most species were collected in temperate (54\%) and tropical (43.2\%) 157 regions. 2.8\% came from dry and continental regions combined (Fig. 3b). We did not consider 158 the original geographic distribution of those species obtained from breeding cultures.

159 A total of 13 biting-chewing insect orders are present in the database (Fig. 3d). We could not 160 obtain life animals from the orders Zoraptera and Grylloblattodea. Bite force measurements 161 of the few species of Plecoptera, Mecoptera, and Trichoptera that were available failed 162 because no voluntary biting could be elicited in these specimens. We did not attempt measuring available representatives of Psocoptera and the biting-chewing "mandibulate archaic moths" (Lepidoptera: Micropterigoidea) due to their minute size. The assessment of 
phylogenetic coverage of the orders and families showed that most families are represented dashed line in Fig. 3a). Accordingly, bite forces of only a fraction of all insect species were measured so far. Nevertheless, the database exceeds all previous studies combined in species

171 numbers (30-fold in insects, 3.5-fold in amniotes), marking just the beginning of research on

172 this performance trait in the most species-ridge metazoan clade.

\section{Usage Notes}

174 The forceR package ${ }^{27}$ was used to create the insect bite force database, which contains

175 cleaned measurement time series and maximum bite forces of insects. The same package may

176 be used to expand the scarce knowledge on insect bite forces by tackling questions regarding

177 the evolution of bite lengths, frequencies, and bite curve shapes by semi-automatically

178 extracting individual bite curves from these measurements. Additionally, the maximum bite

179 force values presented in Online-only table 1 can be used for a wide range of in-depth studies

180 on the morphological and ecological predictors and macroevolution of this important

181 performance trait in the megadiverse insects.

\section{Code Availability}

183 The R code to convert the raw measurements into the final database and to create all tables

184 and figures used in this publication can be found at https://github.com/Peter-T- 


\section{Acknowledgements}

187 We would like to express our deep gratitude to the numerous people that helped in collecting

188 (J. Dorey (Flinders University, Adelaide, Australia), E. P. Fagan-Jeffries (University of Adelaide,

189 Adelaide, Australia), L. R. Beische, M. Bläser, R. Predel (University of Cologne, Cologne,

190 Germany), A. M. Jansen, L. Hamann (University of Bonn, Bonn, German), M. Giannotta

191 (Australian National University, Adelaide, Australia), Sam Finnie (University of South Bohemia,

192 Ceske Budejovice, Czech Republic) J. Hamann (Refrath, Germany) and identifying (T. Frenzel

193 (University of Koblenz - Landau, Koblenz, Germany), J. Dorey (Flinders University, Adelaide,

194 Australia), D. C. F. Rentz (James Cook University Cairns Campus, Cairns, Australia), S. You Ning,

195 J. Rodriguez Arrieta (Australian National Insect Collection, Canberra, Australia), E. P. Fagan-

196 Jeffries (University of Adelaide, Adelaide, Australia), M. Giannotta, Y. Living' Li (Australian

197 National University, Adelaide, Australia), A. Richter (Friedrich-Schiller-Universität Jena, Jena,

198 Germany)) many of the animals used in this study. R. Plarre (Bundesanstalt für

199 Materialforschung und -prüfung (BAM), Berlin, Germany), L. R. Beische, M. Bläser, R. Predel,

200 T. Weihmann (University of Cologne, Cologne, Germany), S. Büsse (Kiel University, Kiel,

201 Germany), W. Hickler (Gelsenkirchen, Germany), C. J. Schwarz (Bochum, Germany), M.

202 Sebesta (Antstore, Berlin, Germany) and B. and D. Drenske (AntsNature, Berlin, Germany) are

203 thanked for providing life animals from their breeding cultures and/or collection trips free of

204 charge. Collections in Australia were carried out at the Daintree Rainforest Observatory (James

205 Cook University, Australia) and under the permit number PTU19-002400 of the Queensland

206 Parks and Wildlife Service. PTR, MF and AB were supported by the European Research Council

207 (ERC) under the European Union's Horizon 2020 research and innovation program (grant

208 agreement no. 754290, 'Mech-Evo-Insect'). AB and CE were supported by the Deutsche

209 Forschungsgemeinschaft (DFG) under the Individual Research Grants program (grant

210 agreement no. BL 1355/4-1). 


\section{Author contributions}

212 PTR wrote all code, prepared the figures, lead the dataset creation and drafted the manuscript.

213 PTR and $A B$ conceived the study and refined the manuscript. $C E, M F$ and $A B$ contributed to the

214 dataset creation. All authors reviewed the manuscript and gave final approval for publication.

\section{Competing interests}

216 The authors declare no competing interests. 


\section{Figures}
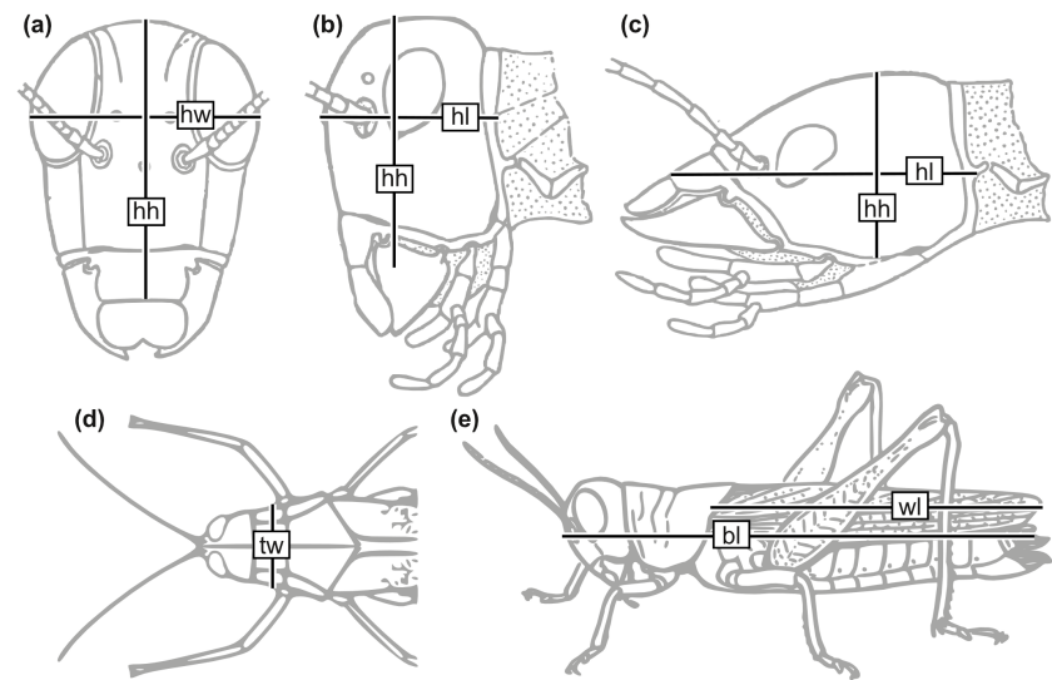

Fig. 1: Insect length measurements. (a-b) Head of an orthognathous insect in frontal (a) and

221 lateral view (b). (c) Head of prognathous insect in lateral view. (d) Frontal part of an 222 orthognathous insect in dorsal view. (e) Orthognathous insect habitus in lateral view.

223 Abbreviations: bl, body length; hh, head height; hl, head length; hw, head width; wt, thorax

224 width; wl, forewing length. a,b,c after Snodgrass ${ }^{42}$; e after Snodgrass ${ }^{43}$. 

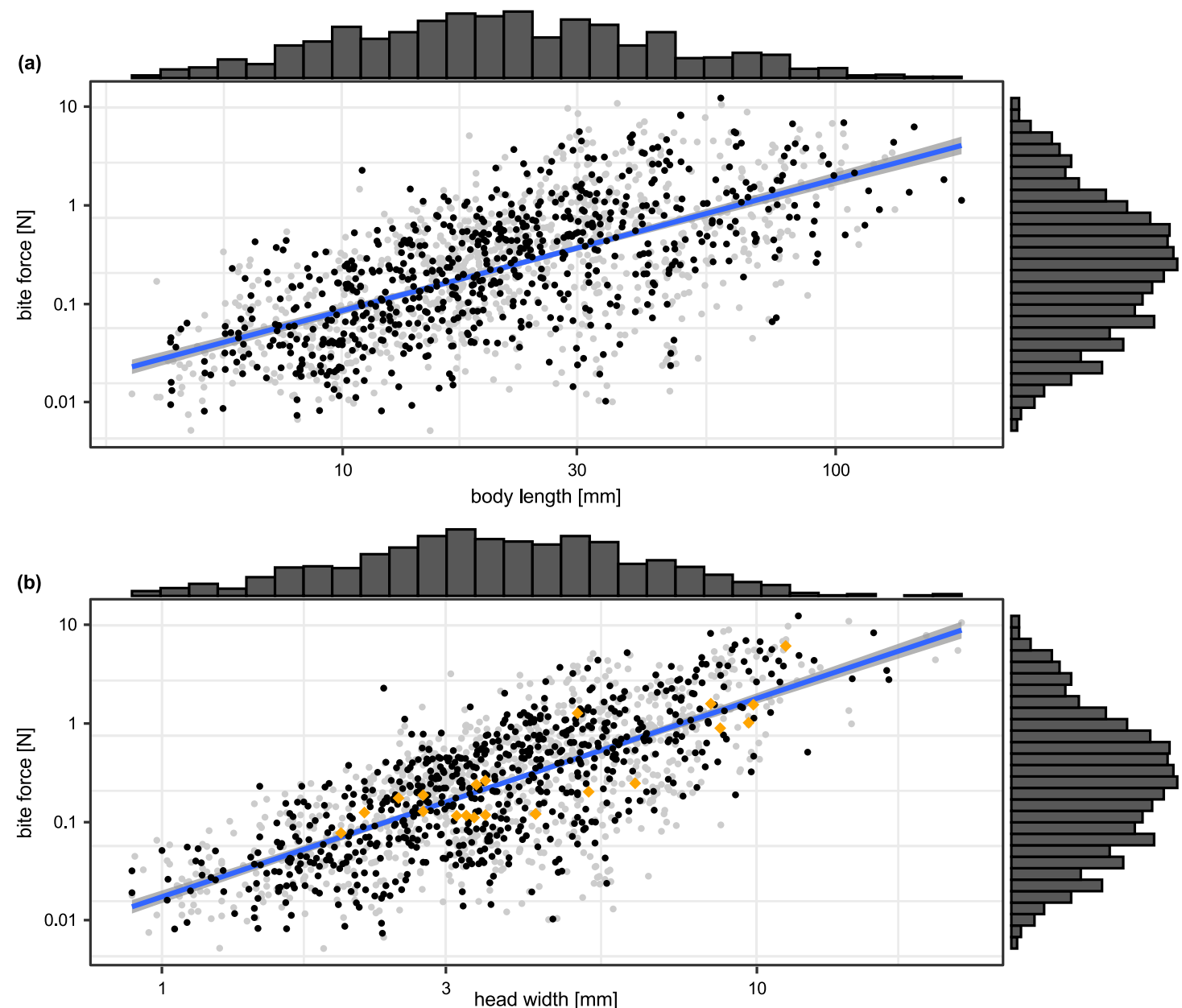

227 Fig. 2: Maximum bite force against body length (a) and head width (b). Grey dots show

228 geometric means of all maximum bite forces per specimen, black dots show geometric means

229 of all length measurements and maximum bite forces of all specimens per species. Marginal

230 histograms at the $x$ - and $y$-axes show mean size and mean bite force distribution per specimen,

231 respectively. Regression lines and coefficients refer to log10-linear models of species-wise bite

232 force against body length (a) or head width (b). Orange diamonds in (b) show bite force

233 measurements available in previous literature. All axes are log10-transformed. 


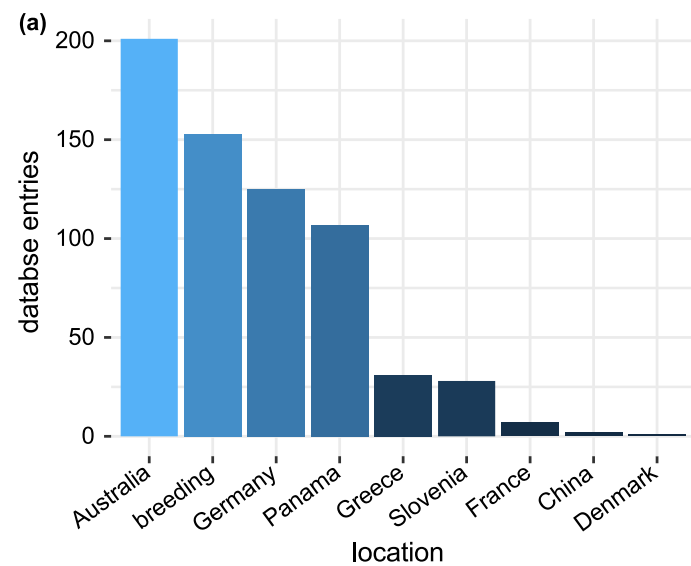

(c)

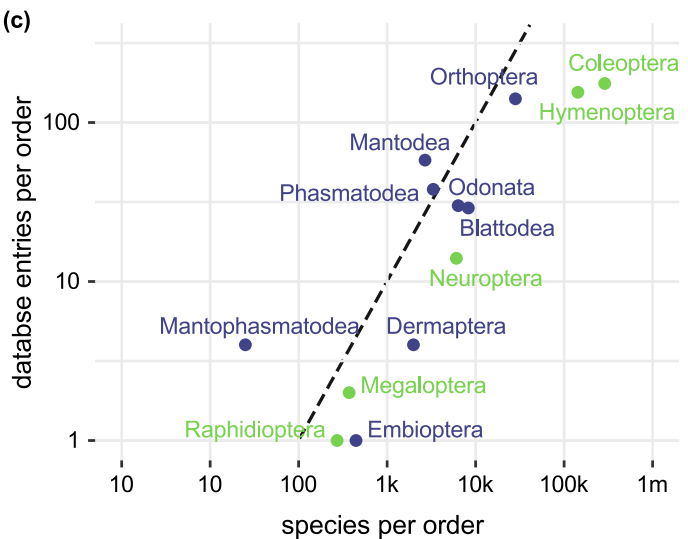

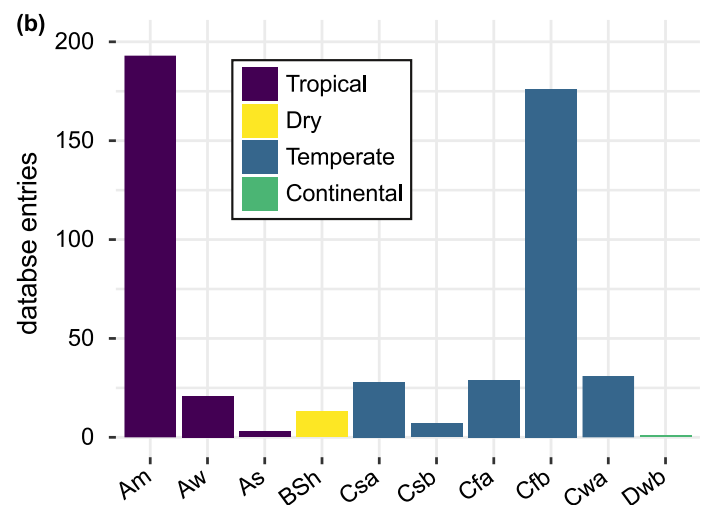

climate zone

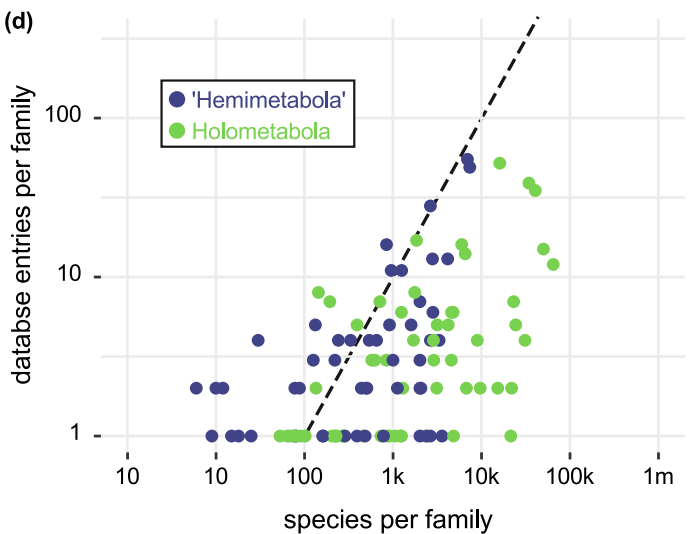

Fig. 3: Geographical and phylogenetic coverage of the bite force database. (a) Species entries

237 per collection location (country or breeding). (b) Species entries per Köppen-Geiger climate

238 zone with specimens sourced from breeding cultures excluded. (c) Ratio of database entries

239 compared to species estimated in all insect orders present in the database. (d) Ratio of

240 database entries compared to species estimated in all insect families present in the database.

241 The dashed lines in $(c, d)$ mark a ratio of 1 data base entry per 100 estimated species.

242 Abbreviations: Am: tropical monsoon; Aw: tropical savanna with dry-winter characteristics;

243 As: tropical savanna with dry-summer characteristics; BSh: semi-arid (steppe) hot; Csa:

244 mediterranean hot summer; Csb: mediterranean warm/cool summer; Cfa: humid subtropical;

245 Cfb: oceanic; Cwa: dry-winter humid subtropical; Dwb: warm summer continental. 


\section{Tables}

249 Online-only Table 1: Insect bite force database summary. Taxonomic classification, maximum

250 bite force per measurement (iBite), specimen and species (ID), regular and geometric mean

251 bite force per specimen and species, voltage amplification setting, length measurements,

252 collection coordinates, country, and climate zone for each bite fore measurement.

253 


\section{References}

255 1. Aguirre, L. F., Anthony, H., van Damme, R. \& Matthysen, E. Ecomorphological analysis of 256 trophic niche partitioning in a tropical savannah bat community. Proc. R. Soc. Lond. B Biol.

257 Sci. 269, 1271-1278 (2002).

258 2. Huyghe, K., Vanhooydonck, B., Scheers, H., Molina-Borja, M. \& Van Damme, R. 259 Morphology, performance and fighting capacity in male lizards, Gallotia galloti. Funct. 260 Ecol. 19, 800-807 (2005).

3. Lappin, A. K. \& Husak, J. F. Weapon performance, not size, determines mating success and potential reproductive output in the collared lizard (Crotaphytus collaris). Am. Nat. 166, 426-436 (2005).

4. Husak, J. F., Lappin, A. K., Fox, S. F. \& Lemos-Espinal, J. A. Bite-Force Performance Predicts Dominance in Male Venerable Collared Lizards (Crotaphytus antiquus). Copeia 2006, 301306 (2006).

5. Meyers, J. J., Nishikawa, K. C. \& Herrel, A. The evolution of bite force in horned lizards: the influence of dietary specialization. J. Anat. 232, 214-226 (2018).

6. Huber, D. R., Eason, T. G., Hueter, R. E. \& Motta, P. J. Analysis of the bite force and mechanical design of the feeding mechanism of the durophagous horn shark Heterodontus francisci. J. Exp. Biol. 208, 3553-3571 (2005).

7. Grubich, J. R., Huskey, S., Crofts, S., Orti, G. \& Porto, J. Mega-Bites: Extreme jaw forces of living and extinct piranhas (Serrasalmidae). Sci. Rep. 2, 1009 (2012).

8. Erickson, G. M. et al. Insights into the Ecology and Evolutionary Success of Crocodilians Revealed through Bite-Force and Tooth-Pressure Experimentation. PLOS ONE 7, e31781 (2012).

9. Herrel, A., Podos, J., Huber, S. K. \& Hendry, A. P. Evolution of bite force in Darwin's finches: a key role for head width. J. Evol. Biol. 18, 669-675 (2005). 
10. Herrel, A., O’Reilly, J. C. \& Richmond, A. M. Evolution of bite performance in turtles. J. Evol. Biol. 15, 1083-1094 (2002).

11. Herrel, A., Grauw, E. D. \& Lemos-Espinal, J. A. Head shape and bite performance in xenosaurid lizards. J. Exp. Zool. 290, 101-107 (2001).

12. Jones, M. E. H. et al. Reproductive phenotype predicts adult bite-force performance in sex-reversed dragons (Pogona vitticeps). J. Exp. Zool. Part Ecol. Integr. Physiol. 333, 252263 (2020).

13. Lappin, A. K. et al. Bite force in the horned frog (Ceratophrys cranwelli) with implications for extinct giant frogs. Sci. Rep. 7, 11963 (2017).

14. Thomason, J. J., Russell, A. P. \& Morgeli, M. Forces of biting, body size, and masticatory muscle tension in the opossum Didelphis virginiana. Can. J. Zool. 68, 318-324 (1990).

15. Wroe, S., Colin, M. \& Jeffrey, T. Bite club: comparative bite force in big biting mammals and the prediction of predatory behaviour in fossil taxa. Proc. R. Soc. B Biol. Sci. 272, 619625 (2005).

16. Christiansen, P. \& Wroe, S. Bite Forces and Evolutionary Adaptations to Feeding Ecology in Carnivores. Ecology 88, 347-358 (2007).

17. Santana, S. E. \& Dumont, E. R. Connecting behaviour and performance: the evolution of biting behaviour and bite performance in bats. J. Evol. Biol. 22, 2131-2145 (2009).

18. Sakamoto, M., Lloyd, G. T. \& Benton, M. J. Phylogenetically structured variance in felid bite force: the role of phylogeny in the evolution of biting performance. J. Evol. Biol. 23, $463-478$ (2010).

19. Labandeira, C. C. Insect mouthparts: Ascertaining the paleobiology of insect feeding strategies. Annu. Rev. Ecol. Syst. 28, 153-193 (1997).

20. Zhang, Z.-Q. Animal Biodiversity: An Outline of Higher-Level Classification and Survey of Taxonomic Richness. Zootaxa 3148, 1-237 (2011). 
21. David, S., Funken, J., Potthast, W. \& Blanke, A. Musculoskeletal modeling of the dragonfly mandible system as an aid to understanding the role of single muscles in an evolutionary context. J. Exp. Biol. jeb.132399 (2016) doi:10.1242/jeb.132399.

22. David, S., Funken, J., Potthast, W. \& Blanke, A. Musculoskeletal modelling under an evolutionary perspective: deciphering the role of single muscle regions in closely related insects. J. R. Soc. Interface 13, 20160675 (2016).

23. Weihmann, T., Reinhardt, L., Weißing, K., Siebert, T. \& Wipfler, B. Fast and Powerful: Biomechanics and Bite Forces of the Mandibles in the American Cockroach Periplaneta americana. PLoS ONE 10, e0141226 (2015).

24. Wheater, C. P. \& Evans, M. E. G. The mandibular forces and pressures of some predacious Coleoptera. J. Insect Physiol. 35, 815-820 (1989).

25. Goyens, J., Dirckx, J., Dierick, M., Van Hoorebeke, L. \& Aerts, P. Biomechanical determinants of bite force dimorphism in Cyclommatus metallifer stag beetles. J. Exp. Biol. 217, 1065-1071 (2014).

26. Grimaldi, D. A. \& Engel, M. S. Evolution of the Insects. (Cambridge University Press, 2005).

27. Rühr, P. T. \& Blanke, A. forceX and forceR: a mobile setup and R package to measure and analyse a wide range of animal closing forces. (in review).

28. Herrel, A., Spithoven, L., van Damme, R. \& de Vree, F. Sexual dimorphism of head size in Gallotia galloti: testing the niche divergence hypothesis by functional analyses. Funct. Ecol. 13, 289-297 (1999).

29. Gomes, V., Herrel, A., Carretero, M. A. \& Kaliontzopoulou, A. New Insights into Bite Performance: Morphological Trade-Offs Underlying the Duration and Magnitude of Bite Force. Physiol. Biochem. Zool. 93, 175-184 (2020). 
30. Lappin, A. K. \& Jones, M. E. H. Reliable quantification of bite-force performance requires use of appropriate biting substrate and standardization of bite out-lever. J. Exp. Biol. jeb.106385 (2014) doi:10.1242/jeb.106385.

31. R Core Team. R: A language and environment for statistical computing. R Foundation for Statistical Computing. (R Foundation for Statistical Computing, 2021).

32. Wickham, H., François, R., Henry, L., Müller, K. \& RStudio. dplyr: A Grammar of Data Manipulation. (2021).

33. Wickham, H. et al. ggplot2: Create Elegant Data Visualisations Using the Grammar of Graphics. (2021).

34. Attali, D. \& Baker, C. ggExtra: Add Marginal Histograms to 'ggplot2', and More 'ggplot2' Enhancements. (2019).

35. Sakamoto, M., Ruta, M. \& Venditti, C. Extreme and rapid bursts of functional adaptations shape bite force in amniotes. Proc. R. Soc. B Biol. Sci. 286, 20181932 (2019).

36. Köppen, W. Das geographische System der Klimate. in Handbuch der Klimatologie (ed. Geiger, R.) 5-44 (Borntraeger, 1936).

37. Rubel, F. \& Kottek, M. Observed and projected climate shifts 1901-2100 depicted by world maps of the Köppen-Geiger climate classification. Meteorol. Z. 135-141 (2010) doi:10.1127/0941-2948/2010/0430.

38. Rubel, F., Brugger, K., Haslinger, K. \& Auer, I. The climate of the European Alps: Shift of very high resolution Köppen-Geiger climate zones 1800-2100. Meteorol. Z. 115-125 (2017) doi:10.1127/metz/2016/0816.

39. Bryant, C., Wheeler, N. R., Rubel, F. \& French, R. H. kgc: Koeppen-Geiger Climatic Zones. (2017).

40. Hinchliff, C. E. et al. Synthesis of phylogeny and taxonomy into a comprehensive tree of life. Proc. Natl. Acad. Sci. 112, 12764-12769 (2015). 
41. Michonneau, F., Brown, J. W. \& Winter, D. J. rotl: an R package to interact with the Open Tree of Life data. Methods Ecol. Evol. 7, 1476-1481 (2016).

42. Snodgrass, R. E. Facts and theories concerning the insect head. Smithson. Misc. Collect. 142, 1-61 (1960). 